\title{
One-Step Analysis of Nonlinear Traveltime Data in Ocean Acoustic Tomography
}

\author{
E. K. SKarsoulis \\ Institute of Applied and Computational Mathematics, Foundation for Research and Technology Hellas, \\ Heraklion, Crete, Greece \\ U. SEND \\ Institut für Meereskunde an der Universität Kiel, Kiel, Germany
}

(Manuscript received 17 April 1998, in final form 3 December 1998)

\begin{abstract}
A new approach based on statistical estimation is proposed for the analysis of tomographic traveltime data in cases of significant nonlinear dependence of the traveltimes on the sound-speed variations. Traditional tomography schemes based on linear perturbative inversions about a single, a priori fixed background state cannot properly handle such cases since the linearized model relations will lead to considerable inversion errors, depending on the extent of nonlinearity. In contrast, the background state is considered here as a variable unknown quantity to be estimated from the traveltime data, simultaneously with the peak identification function and the sound-speed perturbation. Using the maximum likelihood approach and the Gaussian assumption, the statistical estimation problem reduces to a weighted least squares problem to be solved simultaneously for the three unknown quantities. A posteriori inversion-error estimates are derived accounting also for uncertainties in the background selection and the peak identification. The proposed method is applied to nine-month-long traveltime data from the Thetis-2 experiment, conducted from January to October 1994 in the Western Mediterranean Sea, where the variability of the ocean environment gives rise to significant nonlinear dependencies between sound-speed and traveltime variations. The recovered temporal variability and stratification compare well with independent XBT observations.
\end{abstract}

\section{Introduction}

A series of experiments in the past have verified that ocean acoustic tomography introduced by Munk and Wunsch (1979) can successfully map ocean temperature distributions over scales of hundreds (Ocean Tomography Group 1982; Cornuelle et al. 1985; Worcester et al. 1993; Send et al. 1995) to thousands (Dushaw et al. 1993; Cornuelle et al. 1993; Spiesberger and Metzger 1992; Spiesberger et al. 1998) of kilometers. In many tomography experiments conducted up to the present the assumption of small ocean variability about a background state could be made and linear perturbative inversion theory (Franklin 1970; Desaubies 1990; Munk et al. 1995) could be applied.

The ongoing development of global ocean observing systems (GOOS), and the application of data assimilation techniques to general circulation models in recent years has raised the need for systematic monitoring of

Corresponding author address: Dr. E. K. Skarsoulis, Foundation for Research and Technology Hellas, Institute of Applied and Computational Mathematics, PO Box 1527, Heraklion, 71110 Crete, Greece.

E-mail: eskars@iacm.forth.gr the ocean interior on annual to interannual timescales (Munk and Forbes 1989). Some tomography experiments of such long-term nature have been conducted or are currently under way in many parts of the world ocean, such as the Greenland Sea experiment in 198788 (GSP Group 1990; Pawlowicz et al. 1995), the Pacific basin experiment in 1983-89 (Spiesberger et al. 1992), the Thetis-2 experiment in the Western Mediterranean Sea in 1994 (Send et al. 1997; Menemenlis et al. 1997a), the Acoustic Thermometry of Ocean Climate program in the northeastern Pacific Ocean (Munk 1994, 1996; Menemenlis et al. 1997b), and the ongoing multiyear tomography experiment in the Labrador Sea (Marshall et al. 1998).

The monitoring of the ocean interior over long time periods, associated with large sound-speed changes, sets new requirements for the analysis of tomography data. An important issue is that the large ocean variability may give rise to nonlinear dependencies between soundspeed (temperature) and acoustic traveltime variations. This significantly complicates the peak identification and inversion problem. For the solution of the nonlinear inversion problem, Abel transform and iterative methods have been proposed. The Abel transform can be used for nonperturbative ray or modal inversions in the 
range-independent case (Munk and Wunsch 1983; Jones et al. 1986, 1993, 1994), yet its nonlinear nature complicates the use of a priori information. Iterative schemes, on the other hand, enhance the applicability of linear inversion techniques in a straightforward manner (Mercer and Booker 1983; Spiesberger 1985; Cornuelle at al. 1993). Their efficiency is hampered, however, by the computational burden associated with the forward calculations, especially when a broadband wave-theoretic approach is used; furthermore, inversion-error estimates cannot be easily obtained.

A method for simple nonlinear inversions for the total heat content was recently applied to Thetis-2 data (Send 1996b; Send et al. 1997), exploiting information from a single deep-turning ray; a nonlinear relation between arrival time and heat content, derived from historical data, was used to convert a manually obtained peak track into a heat content time series. This approach is straightforward and very efficient, yet it cannot be extended to a larger number of arrivals, which convey information about the vertical structure of the water column.

To analyze four-month-long traveltime data from the 1987 Reciprocal Tomography Experiment, Dushaw et al. (1993) exploited climatological information to a priori determine a time-variable background state (historical mean) to be used as reference for linearization. This approach offers a straightforward way to apply linear inversion methods and relevant statistical theory to nonlinear traveltime data. Nevertheless, year-to-year variability can cause deviations from the climatological mean conditions. Furthermore, in cases where the peak tracking and identification is sensitive to the proper selection of the background state or where the interannual variations give rise to a nonlinear traveltime behavior, the a priori selection may limit the applicability of this method for long-term monitoring systems.

An alternative approach is proposed here by considering the time-variable background state as an unknown to be estimated directly from the tomography data, rather than a priori imposed from the climatology. For this purpose the sound-speed parameter space is discretized in accordance with the anticipated variability and degree of nonlinearity and the background variable is introduced. The problems of background selection, peak identification (association between model peaks and observed peaks), and perturbative inversion are then embedded in a global optimization, maximum likelihood estimation problem. Under the Gaussian hypothesis, this problem reduces to a problem of weighted least squares, which is to be solved simultaneously for the three unknown quantities. Furthermore, inversion-error estimates are derived that also account for the uncertainty in the background selection and the peak identification.

The proposed approach is a generalization of previous works (Send 1996a; Skarsoulis et al. 1996), treating the identification problem in close relation with the inversion procedure for the case of small sound-speed perturbations about a fixed background state. At early stag- es it was attempted to solve for the background state independently from the remaining unknowns (Send 1996b; Skarsoulis 1996), which resulted in estimation overshoots; this problem is remedied here by addressing the global optimization problem.

The contents of the work are organized as follows. The tomographic modeling problem is formulated in section 2 and basic notions are introduced, such as the peak arrivals, the peak identification function, and the sound-speed parameterization, the latter leading to the discretization of the parameter space and the introduction of the background variable. In section 3 the estimation problem is addressed in a statistical framework using the maximum likelihood approach; under the Gaussian assumption estimates are derived for the problem unknowns and the inversion errors. The proposed method is applied to the Thetis-2 experiment. In particular, nine-month-long tomography data are analyzed from the longest $(604.7 \mathrm{~km})$ Thetis-2 transect. A description of the experiment, the data analysis and the comparisons between the inversion results and independent observations are presented in section 4 . A discussion on the capabilities and limitations of the method as well as the main conclusions from the work are contained in section 5 .

\section{Formulation}

\section{a. Peak arrivals}

A standard tomographic setting is considered with a source and receiver at fixed locations in a range-independent ocean. Due to the multipath nature of acoustic propagation in the ocean environment, a pulsed broadband signal emitted by the source will arrive at the receiver as a sequence of peaks (the acoustic arrivals) at different time instants.

The arrival times can be modeled as functionals of the sound-speed profile $c(z)$ by expressing the arrival pattern $a(t ; c)$, defined as the amplitude of the acoustic pressure $p_{r}(t ; c)$ at the receiver in the time domain, through the inverse Fourier transform

$$
\begin{aligned}
a(t ; c) & =\left|p_{r}(t ; c)\right| \\
& =\frac{1}{2 \pi}\left|\int_{-\infty}^{\infty} H_{\mathrm{sr}}(\omega ; c) P_{s}(\omega) e^{j \omega t} d \omega\right|,
\end{aligned}
$$

in terms of the source signal $P_{s}(\omega)$ in the frequency domain and the Green's function $H_{\mathrm{sr}}(\omega ; c)$. The latter depends on the source or receiver locations, the circular frequency $\omega$ and the sound-speed profile $c(z)$. The peak arrivals are defined as the local maxima of the arrival pattern with respect to time (Athanassoulis and Skarsoulis 1995)

$$
\frac{\partial a}{\partial t}\left(\tau_{i} ; c\right)=0, \quad i=1, \ldots, I .
$$

The notion of peak arrivals is generic and encompasses 
ray and modal arrivals as special cases; it can also cope with any modeling approach for the arrival pattern, either ray or wave theoretic. Since the arrival pattern depends on the sound-speed field, the peak arrival times do so as well, that is, $\tau_{i}=\tau_{i}(c)$.

\section{b. Sound-speed parameterization}

The sound-speed variability in a certain area is represented through a modal expansion

$$
c(z)=c_{0}(z)+\sum_{l=1}^{L} \vartheta_{l} \phi_{l}(z)
$$

where $c_{0}(z)$ is a basic reference profile and $\left\{\phi_{l}(z)\right\}$ is a set of sound-speed modes, for example, empirical orthogonal functions (EOFs). The functional dependence of $\tau_{i}$ on the sound speed can be then written as a parametric dependence $\tau_{i}(\boldsymbol{\vartheta})$ on the modal parameter vector $\boldsymbol{\vartheta}=\left\{\boldsymbol{\vartheta}_{l}\right\}, \boldsymbol{\vartheta} \in \Theta$, where $\Theta$ is the parameter space spanning the sound-speed variability expected. Although the dependence $\tau_{i}(\boldsymbol{\vartheta})$ is in general nonlinear, in the case of small variations it can be linearized about a background state $\boldsymbol{\vartheta}=\boldsymbol{\vartheta}_{b}$

$$
\tau_{i}(\boldsymbol{\vartheta})=\tau_{i}\left(\boldsymbol{\vartheta}_{b}\right)+\sum_{l=1}^{L} \frac{\partial \tau_{i}}{\partial \vartheta_{l}}\left(\boldsymbol{\vartheta}_{b}\right)\left[\vartheta_{l}-\vartheta_{b, l}\right] .
$$

The derivatives $\partial \tau_{i} / \partial \vartheta_{l}$ can be analytically expressed in terms of background quantities (Athanassoulis and Skarsoulis 1995), and Eq. (4) can be used as a basis for linear $\boldsymbol{\vartheta}$ inversions of traveltime data (Skarsoulis et al. 1996).

In the case of large sound-speed variations, where the nonlinear dependence of the arrival times on the soundspeed parameters becomes significant, the above linearization about a single background state is insufficient and cannot be used for inversions. Nevertheless, it can be extended in a straightforward manner by considering a set of discrete background states, rather than a single background state, depending on the expected soundspeed variability and the degree of nonlinearity. Then Eq. (4) can be used with respect to the nearest background state each time. To denote the discretization of the parameter space the subscript $b$ will be considered in the following as a variable, $b \in \mathcal{B}$, from the set $\mathcal{B}$ of discrete background states. The background values of the modal parameter vector $\boldsymbol{\vartheta}$ are denoted by

$$
\boldsymbol{\vartheta}_{b} \in \Theta, \quad b \in \mathcal{B} .
$$

Thus the nonlinear relation $\tau_{i}(\boldsymbol{\vartheta})$ is replaced by a set of linear ones [Eqs. (4), (5)]. The cost to be paid for this simplification with respect to the inverse problem is that an additional unknown is introduced: the background variable $b$.

\section{c. Peak identification}

A further unknown, in addition to $b$ and $\boldsymbol{\vartheta}$, arises from the need to associate the model peaks (peak ar- rivals) with the observed peaks before addressing the inversion problem. In particular, the model peak arrival times $\tau_{i}, i=1,2, \ldots, I$ are to be associated with the observed arrival times $\tau_{j}^{(o)}(K), j=1,2, \ldots, J$, in the $K$ th reception $(I \neq J$, in general). This can be done by introducing a one-by-one mapping $g:\{1,2, \ldots, I\} \rightarrow$ $\{1,2, \ldots, J\}$, which is called the identification function (Mauuary 1994; Skarsoulis et al. 1996). Its domain of definition $\mathcal{D}_{g}$ is in general a subset of $\{1,2, \ldots, I\}$.

The identification set $\mathbf{J}(K)$, that is, the set of all possible identification functions (identifications) in the $K$ th reception, is restricted in various ways to avoid unnatural identifications (Send 1996a; Skarsoulis et al. 1996). A first restriction is to exploit information from the previous step $(K-1)$, for which the identification and inversion have been already completed. The subset $\tilde{\mathbf{J}}_{\varepsilon}(K)$ is introduced

$$
\begin{aligned}
\tilde{\mathbf{J}}_{\varepsilon}(K)=\{\mathcal{J} \in & \mathbf{J}(K):\left|\tau_{\mathcal{g}(i)}^{(o)}(K)-\hat{\tau}_{i}(K-1)\right|<\varepsilon(i), \\
& \left.i \in \mathcal{D}_{g}\right\},
\end{aligned}
$$

where $\hat{\tau}_{i}(K-1)$ denotes the predicted traveltimes from (4), with $b=\hat{b}_{K-1}$ and $\boldsymbol{\vartheta}=\hat{\boldsymbol{\vartheta}}_{K-1}$ estimated in the previous step, whereas $\tau_{g_{(i)}}^{(o)}(K)$ denotes the associated observed arrival times in the present step $K$, according to the trial identification $\mathcal{g}$. In other words, a time window of size $2 \varepsilon(i), i=1, \ldots, I$ is set about each predicted arrival time of the previous step, and all observed peaks of the $K$ th reception lying within this window are considered as possible identifications of the particular peak arrival.

If a single observed peak lies in the search windows of two or more subsequent peak arrivals, all possible alternatives have to be built associating the observed peak with a single peak arrival each time. The trial identifications are checked for monotonicity of the arrival times and also for the number of identified peaks in order to keep only those identifying the maximum possible number $M$ of peaks $(M \leq I)$. The resulting set of trial identifications is denoted by $\mathbf{J}_{\varepsilon}(K)$.

\section{Estimation}

The problem of estimating $b, \boldsymbol{\vartheta}$, and $\mathcal{I}$ from the observed traveltimes is addressed in this section. Adopting a statistical setting in which the above quantities are assumed random, hence associated with probabilities or probability densities (Tarantola 1987), and further applying the Bayes formula, the following expression can be obtained for the posterior likelihood of the sought quantities, given the observation vector $\boldsymbol{\tau}_{K}^{(o)}=$ $\left\{\tau_{j}^{(o)}(K)\right\}$ :

$$
\begin{aligned}
\mathcal{L} & \left(b, \mathcal{J}, \boldsymbol{\vartheta} \mid \boldsymbol{\tau}_{K}^{(o)}\right) \\
& =\frac{1}{f_{\tau}\left(\boldsymbol{\tau}_{K}^{(o)}\right)} f_{\tau \mid b, \vartheta, J}\left(\boldsymbol{\tau}_{K}^{(o)} \mid b, \mathcal{J}, \boldsymbol{\vartheta}\right) f_{\vartheta \mid b, J}(\boldsymbol{\vartheta} \mid b, \mathcal{g}) \operatorname{Pr}(b, \mathcal{g}),
\end{aligned}
$$


where $f_{\tau}$ is the probability density function (PDF) of the arrival time vector, whereas $f_{\tau \mid b, \vartheta, J}$ is the conditional PDF of the arrival time vector, given $b, \mathcal{J}$, and $\boldsymbol{\vartheta}$. Further, $f_{\vartheta \mid b, J}$ is the conditional PDF of the parameter vector $\boldsymbol{\vartheta}$, given $b$ and $\mathcal{g}$, and finally $\operatorname{Pr}(b, \mathfrak{g})$ is the probability associated with the particular trial background state and trial identification.

\section{a. A priori information}

The PDFs and the probabilities on the right-hand side of Eq. (7) can be expressed in terms of model relations and a priori statistical information available for the unknown quantities. Starting with $f_{\tau \mid b, \vartheta, J}$, the arrival time prediction (4) is subject to a modeling error (e.g., due to linearization or range-dependent effects); furthermore, the arrival time data are subject to an observational error as well. Considering the difference between predicted and associated observed arrival times,

$$
\begin{gathered}
n_{i}=\tau_{i}\left(\boldsymbol{\vartheta}_{b}\right)+\sum_{l} \frac{\partial \tau_{i}}{\partial \vartheta_{l}}\left(\boldsymbol{\vartheta}_{b}\right)\left[\vartheta_{l}-\vartheta_{b, l}\right]-\tau_{\vartheta_{(i)}^{(o)}}(K), \\
i \in \mathcal{D}_{g},
\end{gathered}
$$

for given $b, \boldsymbol{\vartheta}, \mathfrak{g}$, as a Gaussian, zero-mean random vector, the $\operatorname{PDF} f_{\tau \mid b, \vartheta, J}$ is written in the form

$$
\begin{aligned}
& f_{\tau \mid b, \vartheta, J}\left(\boldsymbol{\tau}_{K}^{(o)} \mid b, \mathcal{J}, \boldsymbol{\vartheta}\right) \\
& \quad=\frac{1}{\sqrt{(2 \pi)^{M} \operatorname{det}_{n n}}} \exp \left\{-\frac{1}{2} \mathbf{n}^{\prime} \mathbf{R}_{n n}^{-1} \mathbf{n}\right\},
\end{aligned}
$$

where $\mathbf{R}_{n n}$ is the covariance matrix of the cumulative observation and modeling error $\mathbf{n}=\left\{n_{i}\right\}$, taken in correspondence with the domain of definition $\mathcal{D}_{\text {g }}$ of the particular trial identification $g$. In the following all vectors are considered as column matrices, whereas a prime denotes matrix transposition.

Concerning the probability density $f_{\vartheta \mid b, J}$, a reasonable choice would be a uniform distribution along the discretization directions, over the domain of validity of each particular background state, and Gaussian distributions along the remaining directions, with variances prescribed from the historical data. However, taking different distributions along different directions would complicate the analysis. For convenience a multivariate Gaussian distribution about $\boldsymbol{\vartheta}_{b}$ is considered:

$$
\begin{aligned}
f_{\vartheta \mid b, J}= & \frac{1}{\sqrt{(2 \pi)^{L} \operatorname{det} \mathbf{R}_{\vartheta \vartheta}}} \\
& \times \exp \left\{-\frac{1}{2}\left(\boldsymbol{\vartheta}-\boldsymbol{\vartheta}_{b}\right)^{\prime} \mathbf{R}_{\vartheta \vartheta}^{-1}\left(\boldsymbol{\vartheta}-\boldsymbol{\vartheta}_{b}\right)\right\},
\end{aligned}
$$

with covariance matrix $\mathbf{R}_{\vartheta \vartheta}$ compatible with the climatology and the discretization. In particular, along the discretization directions, the variance is defined from the discretization step: for example, for uniform discretization of the $\vartheta_{l}$ axis with step $\delta \vartheta_{l}$, the variance is taken equal to $\delta \vartheta_{l}^{2} / 12$, the variance of a uniform distribution in the interval $\left[-\delta \vartheta_{l} / 2, \delta \vartheta_{l} / 2\right]$. The extent of the interval to be discretized is taken in accordance with the historical variance of the corresponding $\vartheta_{l}$ component. For the remaining (nondiscretized) $\boldsymbol{\vartheta}$ components, the historical variance is used.

Regarding the probability $\operatorname{Pr}(b, g)$ in Eq. (7), all $(b, g)$ $\in \mathcal{B} \times \mathbf{J}_{\varepsilon}(K)$ are taken equiprobable since there is no a priori preference for a particular background state and/ or identification function. Finally, the $\operatorname{PDF} f_{\tau}\left(\tau_{K}^{(o)}\right)$ in Eq. (7), no matter what its form may be, is a constant for the particular reception; that is, it plays no role in the estimation process.

\section{b. Maximum likelihood estimation}

Under the above hypotheses the likelihood functional (7) becomes

$$
\begin{aligned}
\mathcal{L}\left(b, \mathcal{I}, \boldsymbol{\vartheta} \mid \boldsymbol{\tau}_{K}^{(o)}\right) & \\
= & \text { constant } \exp \left\{-\frac{1}{2} \mathbf{n}^{\prime} \mathbf{R}_{n n}^{-1} \mathbf{n}\right\} \\
& \times \exp \left\{-\frac{1}{2}\left(\boldsymbol{\vartheta}-\boldsymbol{\vartheta}_{b}\right)^{\prime} \mathbf{R}_{\vartheta \vartheta}^{-1}\left(\boldsymbol{\vartheta}-\boldsymbol{\vartheta}_{b}\right)\right\},
\end{aligned}
$$

for $b \in \mathcal{B}, \mathcal{g} \in \mathbf{J}_{\varepsilon}(K)$, and $\boldsymbol{\vartheta} \in \Theta$. Maximum likelihood estimates $\hat{b}, \hat{g}$, and $\hat{\boldsymbol{\vartheta}}$ can be obtained by maximizing (11) with respect to $b, g$, and $\boldsymbol{\vartheta}$ :

$$
\mathcal{L}\left(\hat{b}, \hat{\mathfrak{g}}, \hat{\boldsymbol{\vartheta}} \mid \boldsymbol{\tau}_{K}^{(o)}\right)=\max _{b, \mathcal{I}, \boldsymbol{\vartheta}} \mathcal{L}\left(b, \mathcal{J}, \boldsymbol{\vartheta} \mid \boldsymbol{\tau}_{K}^{(o)}\right) .
$$

In the case of diagonal covariance matrix $\mathbf{R}_{n n}$ and $\mathbf{R}_{\vartheta \vartheta}$ this is equivalent to minimizing the square sum

$$
\begin{aligned}
S(b, \mathcal{J}, \boldsymbol{\vartheta})= & \sum_{i \in \mathcal{D}_{\mathcal{J}}} \frac{\left[\left\{\mathbf{G}_{b}\left(\boldsymbol{\vartheta}-\boldsymbol{\vartheta}_{b}\right)\right\}_{i}+\tau_{i}\left(\boldsymbol{\vartheta}_{b}\right)-\tau_{\mathcal{g}^{(o)}}^{(o)}(K)\right]^{2}}{\operatorname{diag}\left[\mathbf{R}_{n n}\right]_{i}} \\
& +\sum_{l=1}^{L} \frac{\left(\vartheta-\vartheta_{b, l}\right)^{2}}{\operatorname{diag}\left[\mathbf{R}_{\vartheta \vartheta}\right]_{l}} .
\end{aligned}
$$

The observation matrix $\mathbf{G}_{b}=\left\{\left(\partial \tau_{i} / \partial \vartheta_{l}\right)\left(\boldsymbol{\vartheta}_{b}\right)\right\}$ and the error matrix $\mathbf{R}_{n n}$ in (13) are taken in correspondence with the domain of definition of $\mathcal{D}_{g}$ of the particular trial identification $g$. Thus, the maximum likelihood problem reduces to a weighted least squares problem:

$$
S(\hat{b}, \hat{\mathfrak{g}}, \hat{\boldsymbol{\vartheta}})=\min _{b, \jmath, \boldsymbol{\vartheta}} S(b, \mathcal{g}, \boldsymbol{\vartheta}) .
$$

For fixed $b$ and $g$, the minimization of $S$ with respect to $\boldsymbol{\vartheta}$ can be performed analytically (Skarsoulis et al. 1996), resulting in

$$
\check{\boldsymbol{\vartheta}}(b, \mathcal{g})=\boldsymbol{\vartheta}_{b}+\mathbf{R}_{\vartheta \vartheta} \mathbf{G}_{b}^{\prime}\left(\mathbf{G}_{b} \mathbf{R}_{\vartheta \vartheta} \mathbf{G}_{b}^{\prime}+\mathbf{R}_{n n}\right)^{-1} \delta \boldsymbol{\tau},
$$

where $\delta \boldsymbol{\tau}(b, \mathcal{J} ; K)=\left\{\tau_{\mathscr{g}(i)}^{(o)}(K)-\tau_{i}\left(\boldsymbol{\vartheta}_{b}\right)\right\}_{i \in \mathcal{D}_{\mathfrak{t}}}$. Exploiting (15), the solution of the global optimization problem (14) can be obtained through numerical search in the $\mathcal{B}$ $\times \mathbf{J}_{\varepsilon}(K)$ space. 


\section{c. Inversion-error estimates}

In linear inversion problems, assuming Gaussian statistics, the knowledge of the a priori variance of the model and error vector, $\mathbf{R}_{\vartheta \vartheta}$ and $\mathbf{R}_{n n}$, respectively, and the observation matrix $\mathbf{G}_{b}$ are sufficient for estimating the a posteriori variance of the model vector (Tarantola 1987), that is, for obtaining inversion error estimates. The problem studied here, with unknown background state, model vector, and identification function, as a whole is a nonlinear one. If the background state $b$ and the identification function $\mathcal{I}$ were known, the remaining inversion problem for $\boldsymbol{\vartheta}$ would be linear; in that case the estimate (15) would be the maximum likelihood solution and the a posteriori covariance matrix, for given $\boldsymbol{\tau}$, defined by $\mathbf{D}_{\vartheta \vartheta \mid b j}=\left\langle(\boldsymbol{\vartheta}-\check{\boldsymbol{\vartheta}})(\boldsymbol{\vartheta}-\check{\boldsymbol{\vartheta}})^{\prime}\right\rangle_{\mid b g_{\tau}}$, would be given by

$$
\mathbf{D}_{\vartheta \vartheta \mid b \vartheta}=\left(\mathbf{G}_{b}^{\prime} \mathbf{R}_{n n} \mathbf{G}_{b}+\mathbf{R}_{\vartheta \vartheta}^{-1}\right)^{-1} .
$$

However, both the background state and the identification function are unknowns to be estimated from the tomography data and this fact introduces additional uncertainty. The a posteriori probability density of $\boldsymbol{\vartheta}$, for given $\boldsymbol{\tau}$, can be calculated in this case as the marginal distribution of the joint likelihood function with respect to $b$ and $g$ :

$$
f_{\vartheta \mid \tau}(\boldsymbol{\vartheta} \mid \boldsymbol{\tau})=\sum_{b} \sum_{g} f_{\vartheta \mid b g_{\tau}}(\boldsymbol{\vartheta} \mid b, g, \boldsymbol{\tau}) \operatorname{Pr}(b, g \mid \boldsymbol{\tau})
$$

In the following the quantities on the right-hand side of (17) are expressed analytically under the Gaussian assumption. In this case, the conditional probability density $f_{\vartheta \mid b g_{\tau}}(\boldsymbol{\vartheta} \mid b, \mathcal{J}, \boldsymbol{\tau})$ is a Gaussian distribution as well (Skarsoulis et al. 1996) with mean value $\check{\vartheta}(b, g)$ and covariance matrix $\mathbf{D}_{\vartheta \vartheta \mid b g}$, that is,

$$
\begin{aligned}
& f_{\vartheta \mid b g_{\tau}}(\boldsymbol{\vartheta} \mid b, g, \boldsymbol{\tau}) \\
& \quad=\text { constant } \exp \left\{-\frac{1}{2}(\boldsymbol{\vartheta}-\check{\boldsymbol{\vartheta}})^{\prime} \mathbf{D}_{\vartheta \vartheta}^{-1}(\underline{\jmath} \mid b(\boldsymbol{\vartheta}-\check{\boldsymbol{\vartheta}})\} .\right.
\end{aligned}
$$

The a posteriori probability $\operatorname{Pr}(b, g \mid \boldsymbol{\tau})$ can be expressed taking the marginal of the likelihood function (11) with respect to $\boldsymbol{\vartheta}$ :

$$
\operatorname{Pr}(b, g \mid \boldsymbol{\tau})=\frac{1}{C} \int_{\Theta} \mathcal{L}(b, \mathcal{g}, \boldsymbol{\vartheta} \mid \boldsymbol{\tau}) d \boldsymbol{\vartheta},
$$

where $C$ is a normalizing constant defined by

$$
C=\sum_{b} \sum_{g} \int_{\Theta} \mathcal{L}(b \mathcal{g}, \boldsymbol{\vartheta} \mid \boldsymbol{\tau}) d \boldsymbol{\vartheta},
$$

such that $\Sigma_{b} \Sigma_{g} \operatorname{Pr}(b, g \mid \tau)=1$. Using (11) the above integral over $\boldsymbol{\vartheta}$ can be calculated in closed form:

$$
\begin{aligned}
\int_{\Theta} \mathcal{L}(b, \mathcal{J}, \boldsymbol{\vartheta} \mid \boldsymbol{\tau}) d \boldsymbol{\vartheta} \\
=\operatorname{constant}\left(\frac{\operatorname{det} \mathbf{D}_{\vartheta \vartheta \mid b \jmath}}{\operatorname{det} \mathbf{R}_{n n}}\right)^{1 / 2} \\
\times \exp \left\{\frac{1}{2}\left(\check{\boldsymbol{\vartheta}}-\boldsymbol{\vartheta}_{b}\right)^{\prime} \mathbf{D}_{\vartheta \vartheta \mid b g}^{-1}\left(\check{\boldsymbol{\vartheta}}-\boldsymbol{\vartheta}_{b}\right)\right. \\
\left.\quad-\frac{1}{2}\left(\boldsymbol{\tau}_{J}^{(o)}-\boldsymbol{\tau}_{b}\right)^{\prime} \mathbf{R}_{n n}^{-1}\left(\boldsymbol{\tau}_{J}^{(o)}-\boldsymbol{\tau}_{b}\right)\right\},
\end{aligned}
$$

where $\boldsymbol{\tau}_{J}^{(o)}=\left\{\tau_{g_{(i)}^{(o)}}\right\}$ and $\boldsymbol{\tau}_{b}=\left\{\tau_{i}\left(\boldsymbol{\vartheta}_{b}\right)\right\}, i \in \mathcal{D}_{g}$. Substituting the above Eqs. (18)-(21) into (17) a closed form expression can be obtained for $f_{\vartheta \mid \tau}(\boldsymbol{\vartheta} \mid \boldsymbol{\tau})$. The resulting distribution is a superposition of Gaussian distributions with different mean values and covariance matrices. Defining the global a posteriori covariance matrix as

$$
\begin{aligned}
\mathbf{D}_{\vartheta \vartheta} & =\left\langle(\boldsymbol{\vartheta}-\hat{\boldsymbol{\vartheta}})(\boldsymbol{\vartheta}-\hat{\boldsymbol{\vartheta}})^{\prime}\right\rangle \\
& =\int_{\Theta} f_{\vartheta \mid \tau}(\boldsymbol{\vartheta} \mid \boldsymbol{\tau})(\boldsymbol{\vartheta}-\hat{\boldsymbol{\vartheta}})(\boldsymbol{\vartheta}-\hat{\boldsymbol{\vartheta}})^{\prime} d \boldsymbol{\vartheta}
\end{aligned}
$$

and substituting the expression (17), $\mathbf{D}_{\vartheta \vartheta}$ can be finally expressed as

$$
\begin{aligned}
\mathbf{D}_{\vartheta \vartheta}= & \sum_{b} \sum_{g} \operatorname{Pr}(b, g \mid \boldsymbol{\tau}) \\
& \times\left[\mathbf{D}_{\vartheta \vartheta \mid b \mathcal{g}}+(\check{\boldsymbol{\vartheta}}-\hat{\boldsymbol{\vartheta}})(\check{\boldsymbol{\vartheta}}-\hat{\boldsymbol{\vartheta}})^{\prime}\right] .
\end{aligned}
$$

This is the global covariance matrix accounting for the cumulative error due to the linear inversion, the background state, and the identification function uncertainty. If the background state and identification function are known, $\hat{\boldsymbol{\vartheta}}$ and $\mathbf{D}_{\vartheta \vartheta}$ reduce to $\breve{\boldsymbol{\vartheta}}$ and $\mathbf{D}_{\vartheta \vartheta \mid b g}$, respectively.

Using the parametric expression (3), the following result is obtained for the a posteriori variance $\left\langle\delta c^{2}(z)\right\rangle$ $=\left\langle[c(z)-\hat{c}(z)]^{2}\right\rangle$ of the sound speed

$$
\left\langle\delta c^{2}(z)\right\rangle=\operatorname{diag}\left[\boldsymbol{\phi}^{\prime}(z) \mathbf{D}_{\vartheta \vartheta} \boldsymbol{\phi}(z)\right],
$$

where $\boldsymbol{\phi}(z)=\left\{\phi_{l}(z)\right\}$ is the vector containing the values of the sound-speed modes at different depths. Taking depth averages over particular depth classes the corresponding averaged sound-speed variance becomes $\left\langle\delta \bar{c}^{2}\right\rangle$ $=\overline{\boldsymbol{\phi}}^{\prime} \mathbf{D}_{\vartheta \vartheta} \overline{\boldsymbol{\phi}}$, where the depth-averaged sound speed and sound-speed modes are denoted by overbars.

\section{Application}

The method described in the previous sections is used in the following to perform range-independent inversions of nine-month-long tomography data from the Thetis-2 experiment. In particular, the longest (604.7 $\mathrm{km}$ ) transect, W3-H, is considered for which systematic XBT measurements are also available. 


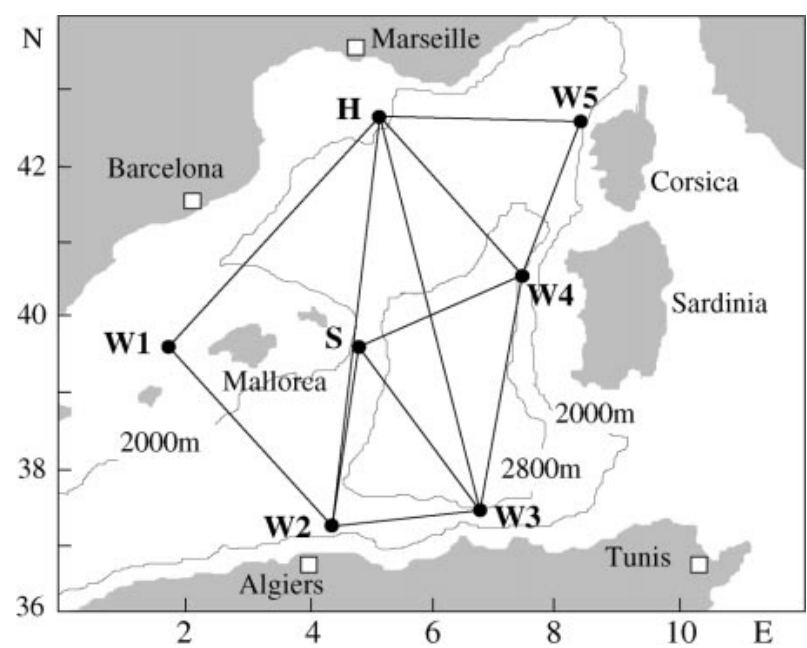

FIG. 1. The geometry of the Thetis-2 experiment in the Western Mediterranean Sea.

\section{a. The Thetis-2 experiment}

The Thetis-2 experiment was conducted from January to October 1994 in the Western Mediterranean Sea (Send 1996b; Send et al. 1997), as a joint effort of IfM (Institut für Meereskunde, Kiel, Germany), IFREMER (Institut Français de Recherche pour l'Exploration de la Mer, Brest, France), IACM (Institute for Applied and Computational Mathematics, Heraklion, Greece), COM (Centre d'Oceanologie de Marseille, La Seyne-sur-Mer, France), and WHOI (Woods Hole Oceanographic Institution, Woods Hole, Massachusetts).

Figure 1 shows the experimental site and geometry. The tomographic array contained seven moored transceivers all deployed at a nominal depth of $150 \mathrm{~m}$. An HLF-5 source, marked by $\mathrm{H}$ in Fig. 1, with a central frequency of $250 \mathrm{~Hz}$ and effective bandwidth of 62.5 $\mathrm{Hz}$, insonified the basin at 8-h intervals. The remaining six sources (W1-W5 and S), transmitting six times per day, were of the Webb type with a central frequency $400 \mathrm{~Hz}$ and an effective bandwidth $100 \mathrm{~Hz}$. The receiver parts of W1-W5 were modified to listen to both the $400-$ and $250-\mathrm{Hz}$ signals. Along the section W3-H an XBT verification line was occupied every two weeks using a commercial vessel connecting Marseille (France) to Skikda (Algeria). In particular, the positions of $\mathrm{H}$ and $\mathrm{W} 3$ were aligned with the ship's route to allow detailed comparisons between tomographic inversions and XBT data. Tomography data from this transect (receptions at W3 from $\mathrm{H}$ ) spanning the period from 24 January to 16 October 1994 (yeardays 24.4-289.4) are analyzed below.

During the deployment and recovery cruises, carried out with the FS Poseidon (IfM) and NO Le Suroit (IFREMER), respectively, hydrographic temperature and salinity profiles were collected over the area of interest to be used in the tomographic analysis for initialization and calibration purposes. The CTD sound- (a) Poseidon, yeardays 14, 27-32

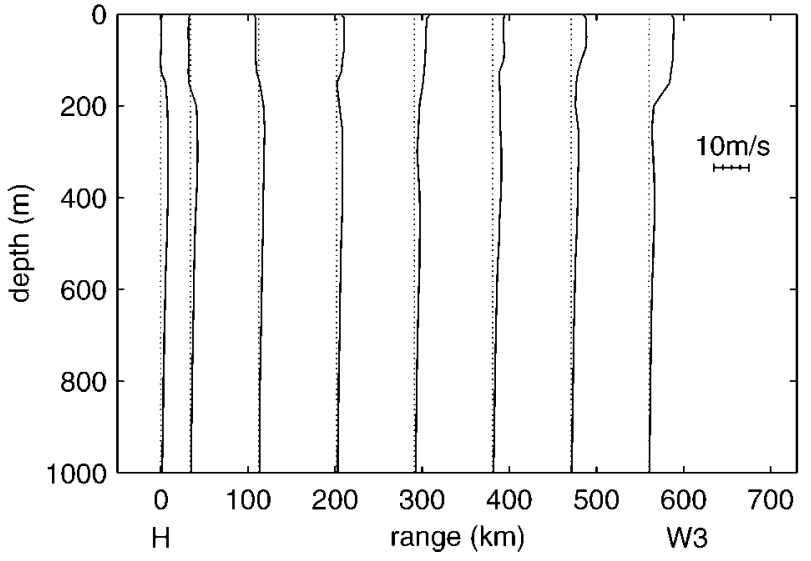

(b) Le Suroit, yeardays 289-292

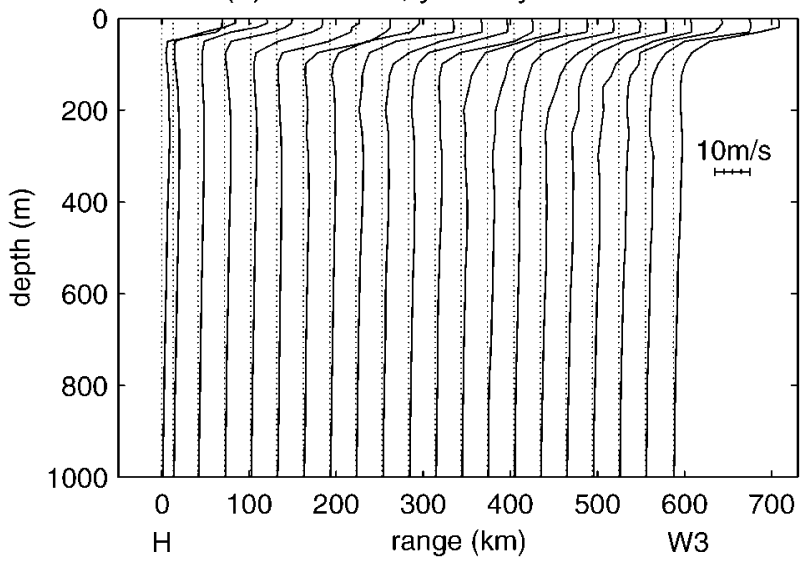

FIG. 2. CTD sound-speed profiles (deviations from a linear reference profile: $1503 \mathrm{~m} \mathrm{~s}^{-1}$ at the surface and $1546.9 \mathrm{~m} \mathrm{~s}^{-1}$ at $2500-\mathrm{m}$ depth) measured along the section W3-H. (a) Poseidon cruise, Jan 1994. (b) Le Suroit cruise, Oct 1994.

speed data from the Poseidon and Le Suroit cruises along W3-H are shown in Fig. 2 in the form of deviations from a linear sound-speed profile $\left(1503 \mathrm{~m} \mathrm{~s}^{-1}\right.$ at the surface and $1546.9 \mathrm{~m} \mathrm{~s}^{-1}$ at 2500-m depth), the latter corresponding to fully mixed conditions, typical for the (northern) Mediterranean in winter. The poor horizontal sampling of the Poseidon section is due to adverse weather conditions encountered in January. Note in Fig. 2 that there is a warming gradient from north to south, due to which the medium properties in the upper 300 $\mathrm{m}$ are dependent on both range and depth. Furthermore, there are large seasonal changes from the Poseidon to Suroit conditions. The Poseidon profiles are close to linear, especially in the northern part of the basin (close to $\mathrm{H}$ ), corresponding to homogeneous fully mixed conditions. In the Suroit section the surface layer is significantly warmer and the resulting sound-speed profiles exhibit a minimum (channel axis) at about 100-m depth. The difference between the Poseidon and Suroit rangeaveraged sound speed reaches $20 \mathrm{~m} \mathrm{~s}^{-1}$ at the surface. 


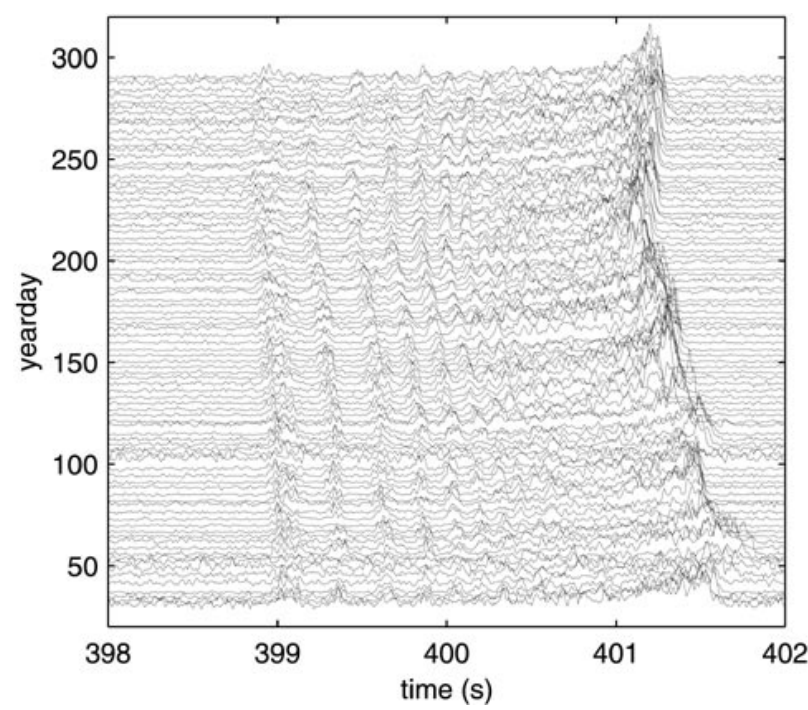

FIG. 3. The acoustic data (3-day incoherently averaged arrival patterns) recorded at $\mathrm{W} 3$ from $\mathrm{H}$ after correlation processing, clockdrift, and mooring motion correction.

\section{b. Data preprocessing}

Figure 3 shows the acoustic receptions of the source $\mathrm{H}$ at $\mathrm{W} 3$ after correlation (matched filter) processing, clock-drift and mooring-motion corrections (Send 1996b); in particular, the 3-day incoherently averaged arrival patterns are shown in this figure. It is seen that the early arrivals at W3 are raylike; five to six ray groups can be distinguished in most receptions. The remaining intermediate and late arrivals are difficult to interpret in terms of ray arrivals because ray groups overlap with each other in this interval. To exploit the maximum of information contained in the intermediate and late part of the arrival patterns, the notion of peak arrivals is used combined with normal-mode propagation modeling.

An offset correction has to be applied to the data to account for the fact that the mean horizontal distance between the moorings is not known accurately enough. This correction can be fixed by comparing the predicted arrival patterns [based on the CTD sound-speed data available (Fig. 2) and the nominal instrument positions] with the corresponding acoustic data. Prior to the acoustic predictions the Poseidon and Suroit sound-speed data are subjected to the earth-flattening transformation (Aki and Richards 1980; Spiesberger and Metzger 1991) to account for the earth curvature effects:

$$
\begin{aligned}
z^{\prime} & =z\left(1+\frac{\epsilon}{2}+\frac{\epsilon^{2}}{3}\right) \text { and } \\
c^{\prime}\left(z^{\prime}\right) & =c(z)\left(1+\epsilon+\epsilon^{2}\right),
\end{aligned}
$$

where $\epsilon=z / R_{0}$ and $R_{0}$ is the earth radius, which equals $6371 \mathrm{~km}$. The above transformation is accurate up to the second order with respect to $\epsilon$.

The comparisons between the measured and the pre- (a) Poseidon, yeardays 14, 27-32

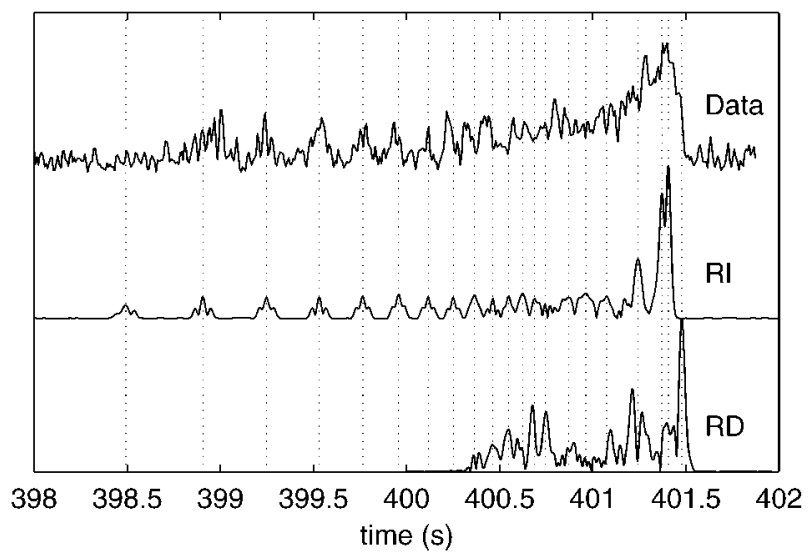

(b) Le Suroit, yeardays 289-292

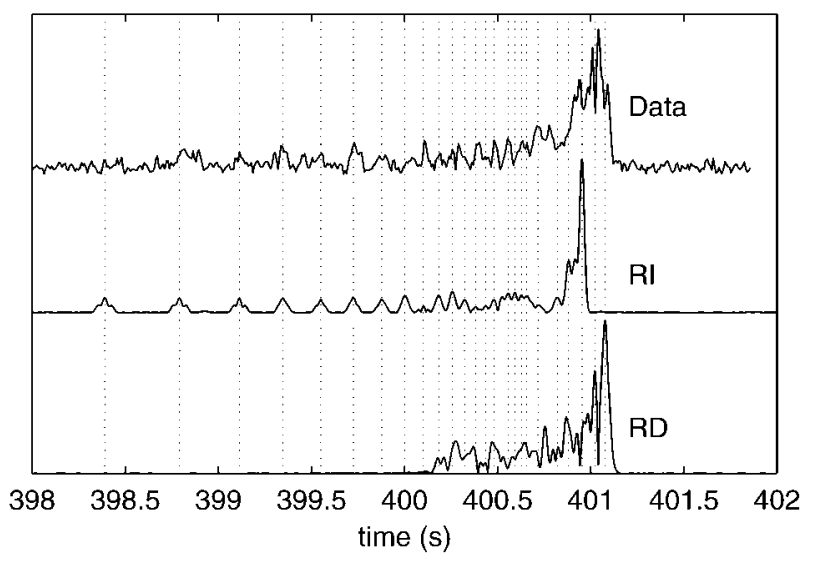

FIG. 4. Comparison of range-independent (RI) and range-dependent (RD) predictions of arrival patterns at W3 from $\mathrm{H}$ using the Poseidon and Suroit CTD sound-speed sections (Fig. 2) with the corresponding offset-calibrated acoustic data.

dicted arrival patterns are shown in Fig. 4 after offset calibration of the tomography data by $-127 \mathrm{~ms}$, corresponding to an offset in the horizontal mooring distance by about $-190 \mathrm{~m}$. The tomography data shown are the incoherent averages of the arrival patterns measured during or close to the period of the CTD samplings. The predictions are performed with the KRAKEN normal/coupled-mode code (Porter and Reiss 1984) assuming the emitted signal to be a Gaussian pulse with a central frequency of $250 \mathrm{~Hz}$ and bandwidth of $62.5 \mathrm{~Hz}$. Both range-independent (RI) and rangedependent/coupled-mode (RD) predictions are shown. For the RI predictions, range-averaged profiles are calculated from the original sections. In the RD case only the first 20 propagating modes are taken into account, corresponding to the intermediate and late part of the arrival pattern, where the RD effect is significant, in order to reduce the computational burden.

It is seen in Fig. 4 that the RI prediction of the early arrivals is in good agreement with the measured data in both the Poseidon and Suroit cases. This is expected 

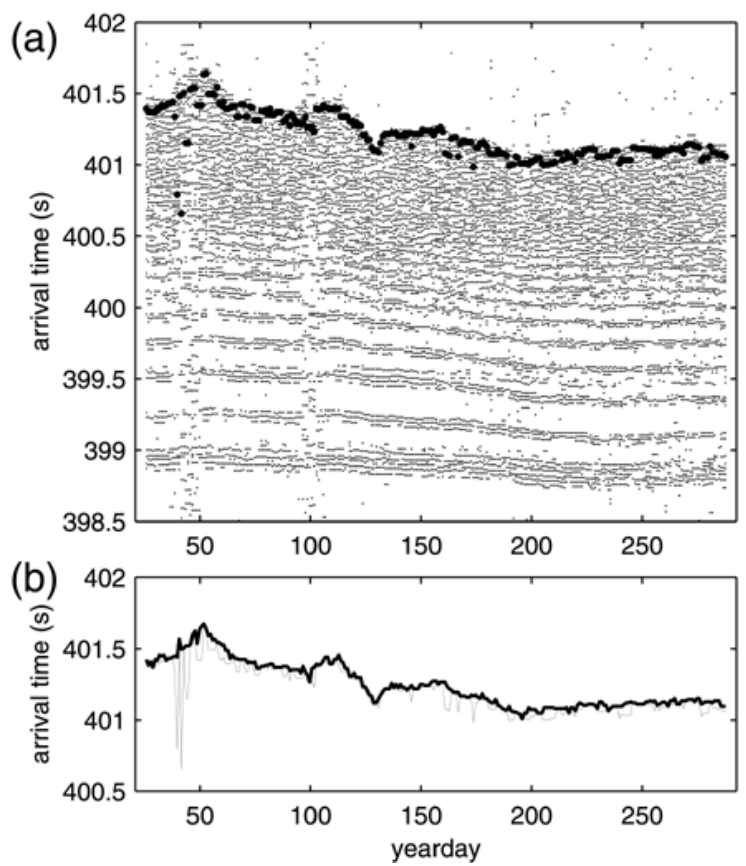

Fig. 5. Measured acoustic arrival times at $\mathrm{W} 3$ from $\mathrm{H}$ over the 9-month duration of the experiment (3-day sliding averages of the arrival patterns are used). (a) Peak locations (light dots) and highest peak locations (heavy dots) in each reception. (b) Highest (light) and cutoff (heavy) peak arrival times.

since early arrivals correspond to steep rays, spending most of their travel time in the deep water layers where the medium is practically range independent. The rangedependence effect becomes gradually more important for the later arrivals sampling shallower layers. The strongest effect is in the final cutoff time, where the difference between the RI and RD prediction ranges from $75 \mathrm{~ms}$ in the Poseidon case to $120 \mathrm{~ms}$ in the Suroit case. The cutoff times of the measured data are reproduced through the RD prediction to within $20-30 \mathrm{~ms}$ in both cases, while the RI calculations predict earlier cutoff times by $60-140 \mathrm{~ms}$.

Figure 5 shows the offset calibrated W3-H acoustic data in the form of observed peak arrival times. The horizontal axis of this figure represents yeardays of 1994 and spans the period of the experiment, whereas the vertical axis measures arrival time. To increase the statistical significance of the data 3-day sliding averages are used; that is, for each calendar day the arrival patterns measured from the preceding to the following day are incoherently averaged. The 45-50 more significant peaks in each reception are shown through dots in the top panel of Fig. 5; the highest peaks are denoted by heavier dots in this figure. Six early arrival groups can be distinguished over the period of the experiment. Later groups of arrivals get closer to each other and cannot be easily resolved over all periods. The bottom panel in Fig. 5 shows the evolution of the highest and the cutoff peaks, the latter being defined as the last of the
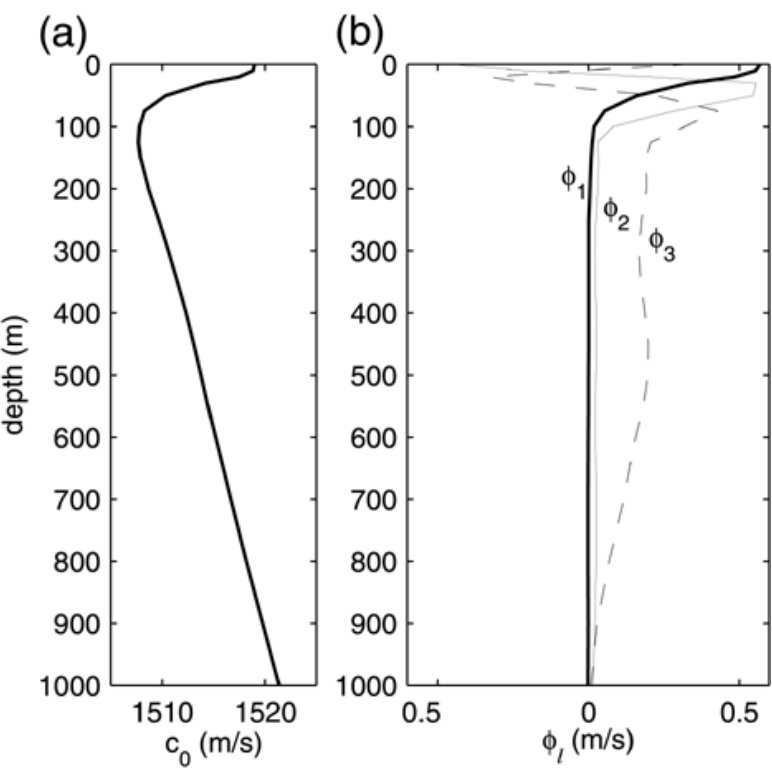

FIG. 6. (a) The basic reference sound-speed profile and (b) the first three EOFs for the western Mediterranean basin.

five highest peaks in each reception. The evolution of the cutoff peaks is significantly more stable than that of the highest peaks that exhibit a significant variability. In this connection, the cutoff peaks are considered to be more reliable for performing inversions.

\section{c. Data analysis}

To prepare the framework for inversions a number of steps have to be undertaken concerning the establishment of sound-speed modes and a priori statistical information, the discretization of the parameter space (definition of the background states), and the calculation of the observation matrix, that is, the derivatives of the peak-arrival times with respect to the sound-speed modes at each background state.

Principal value analysis of historical data for the western Mediterranean basin resulted in a series of EOFs, the first three of which are shown in Fig. 6, along with the basic reference profile. The corresponding rms values of the EOF amplitudes are $\vartheta_{1, \mathrm{rms}}=18.48, \vartheta_{2, \mathrm{rms}}=$ 2.88 , and $\vartheta_{3, \text { rms }}=0.96$. EOF-1 accounts for the bulk of the seasonal sound-speed changes taking place close to the surface, whereas higher-order EOFs extend to increasingly deeper layers. The first three EOFs explain $99.6 \%$ of the total variance. Since the large sound-speed variations are mainly due to EOF-1 representing the seasonal changes, a one-dimensional discretization of the parameter space is considered in the following by defining the background states as EOF-1 variations of the basic reference profile in the $\vartheta_{1}$ interval $[-40,40]$ (two standard deviations about the basic reference state) with a step $\delta \vartheta_{1}=2$. Following section 3a, the variances used for $\mathbf{R}_{\vartheta \vartheta}$ are $4 / 12,2.88^{2}$, and $0.96^{2}$ for EOFs 1, 2, 

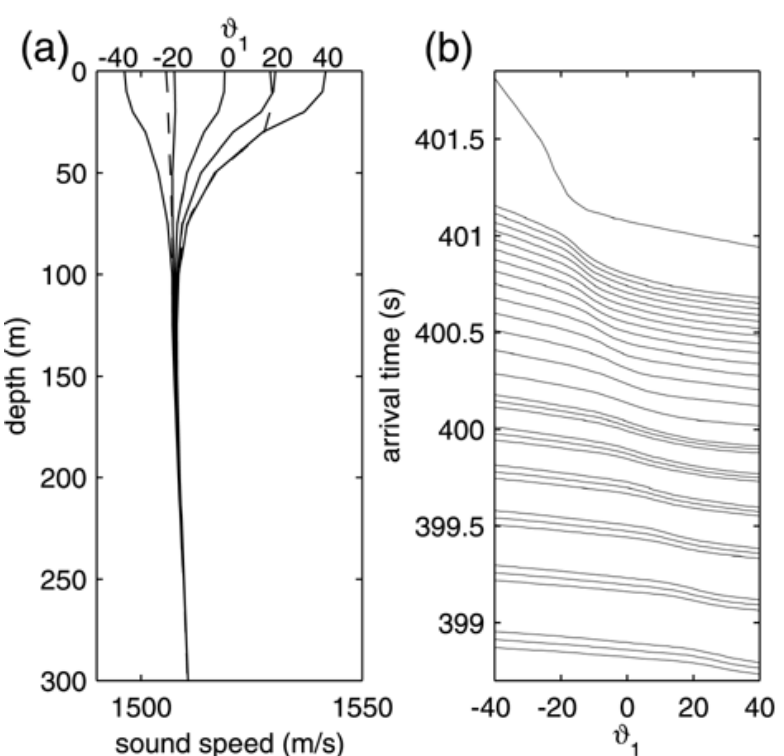

FIG. 7. (a) The background sound-speed profiles for $\vartheta_{1}=-40$, $-20,0,20$, and 40 (solid lines) and the range-averaged Poseidon, Suroit profiles (dashed lines). (b) The background peak arrival times as functions of $\vartheta_{1}$.

and 3 , respectively. Figure 7a shows the dependence of the background sound-speed profiles on $\vartheta_{1}$, ranging from an upward-refracting profile for $\vartheta_{1}=-40$ to a channeled one for $\vartheta_{1}=40$. The Poseidon and Suroit range-averaged profiles are also shown in this figure through dashed lines.

In Fig. $7 \mathrm{~b}$ the background peak-arrival times are shown over the $\vartheta_{1}$ interval $[-40,40]$. In total, 33 model peaks are traced over the considered range of variability. It is seen in this figure that there are $\vartheta_{1}$ intervals of nonlinearity that are different for the different peaks; earlier peaks exhibit the nonlinear behavior for larger $\vartheta_{1}$ values than later peaks. Furthermore, the arrival time variability is larger for the late peaks than for the early ones. The underlying reason for the nonlinear behavior is the passage from surface-reflected propagation in winter to channeled propagation in summer. In terms of ray theory, this passage takes place when the sound-speed value at the surface equals the value at the ray up-turning depth; this value is larger for deep rays, which correspond to early arrivals, than for shallower rays, which correspond to late arrivals. Accordingly, in terms of $\vartheta_{1}$, the transition will take place at larger $\vartheta_{1}$ values for early arrivals than for late ones. This explains the different intervals of nonlinearity for the different peaks in Fig. $7 \mathrm{~b}$.

The observation matrix $\mathbf{G}_{b}$ is calculated for the 33 peak arrivals of Fig. $7 \mathrm{~b}$ at each background state using the KRAKEN normal-mode code; it is shown in Fig. 8, for three background states $\left(\vartheta_{1}=-40,0,40\right)$. The horizontal axis in this figure serves for identification of the peak arrivals by traveltime, whereas the influence coefficients are measured on the vertical axis; points
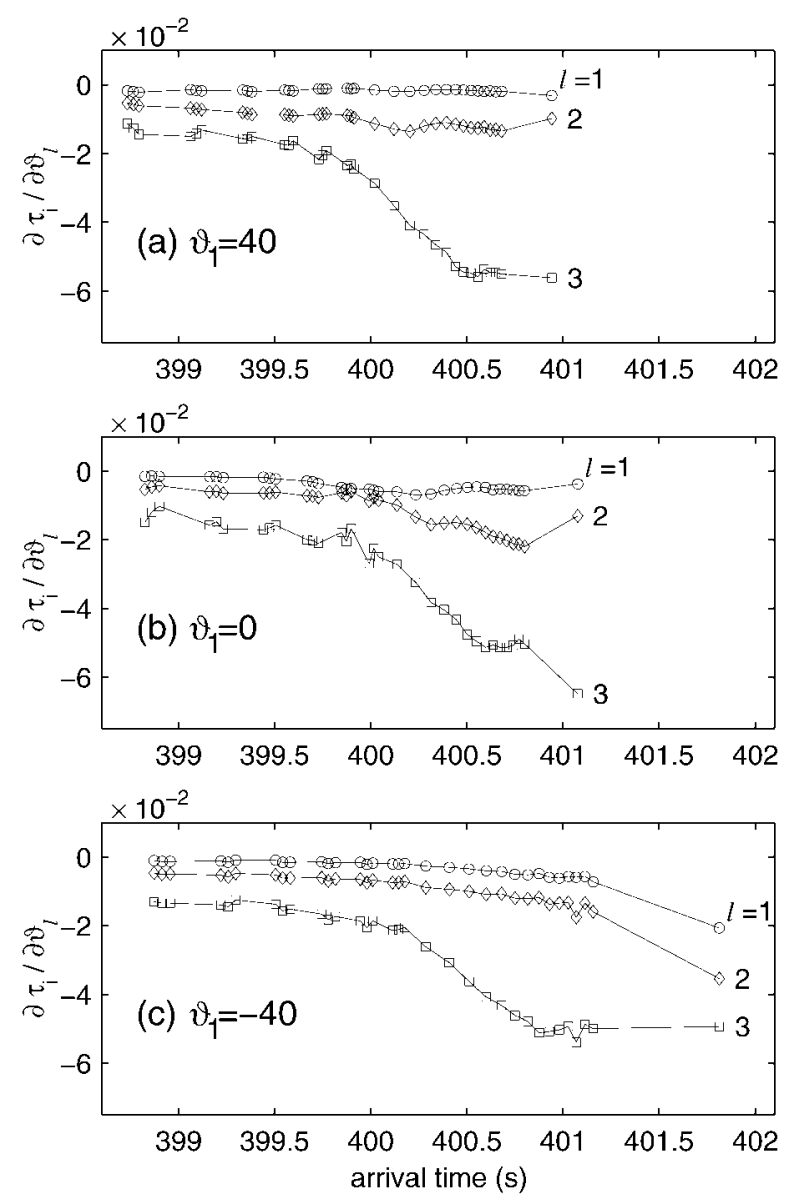

FIG. 8. The observation (influence) matrix for the 33 peak arrivals calculated at three background states $\vartheta_{1}=-40,0$, and 40 for EOF- 1 $(\bigcirc)$, EOF-2, $(\diamond)$ and EOF-3 ( $\square)$.

corresponding to the same EOF are connected with lines. Note that each EOF has a different effect on the peak arrival times and this effect is also dependent on the background state considered; for example the effect of EOF-1 on the last peak arrival is stronger for $\vartheta_{1}=$ -40 than for $\vartheta_{1}=40$, and this can be seen in Fig. 7b as well.

The last quantities to be set prior to the inversion is the error covariance matrix $\mathbf{R}_{n n}$ and the search windows $\varepsilon$ to be used for the construction of the identification set. The data errors are given rms values of $10 \mathrm{~ms}$ for the first six arrival groups, that is, for the first 18 peak arrivals, for which the raylike triplet character is quite clear in the data. For the next arrivals an increasingly larger rms error- $20 \mathrm{~ms}$ for the first six arrivals and 30 ms for the next eight-is taken to account for the increasing uncertainty in the data (no clear triplets present) and the larger expected prediction error. The last peak arrival is associated with the cutoff peak in the data, defined as the last of the five highest peaks in each reception. As seen in the previous section, the last predicted peak is subject to a significant range-dependent 


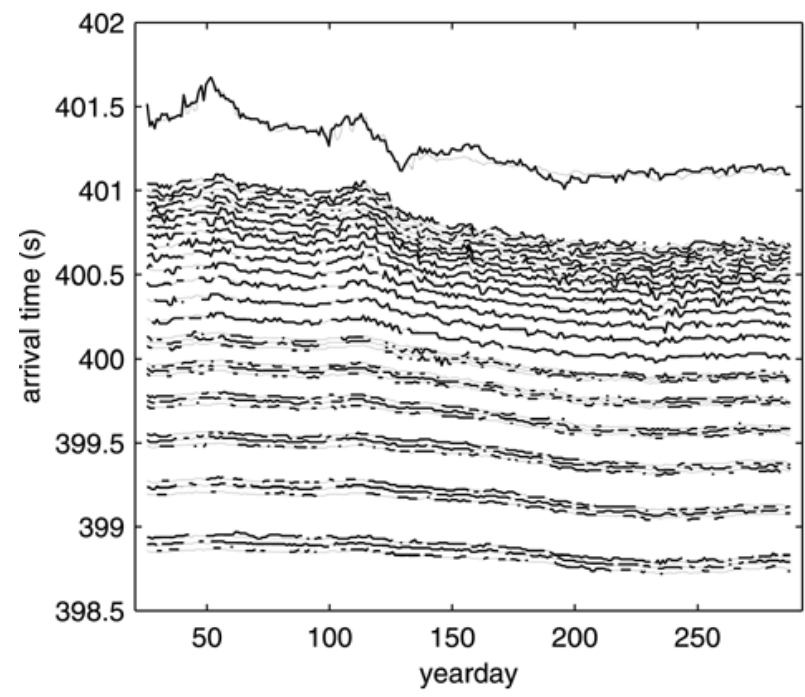

FIG. 9. Identified peak tracks in the acoustic data received at W3 from $\mathrm{H}$ (heavy lines) and predicted peak arrival times (light lines).

effect, which has to be taken into account in the following range-independent inversions (Cornuelle et al. 1985; Munk and Wunsch 1985). In appendix A it is shown that the anticipated range-dependence of the particular environment causes a systematic delay on the last predicted arrival, by $100 \mathrm{~ms}$ in the mean. After correcting for this effect the observation error for the last arrival is taken as $50 \mathrm{~ms}$ rms. For the previous peaks the range-dependent effect lies within the observation error considered for the inversion.

The window radius $\varepsilon$ for determining the trial identifications is set equal to $20 \mathrm{~ms}$ for the first 18 arrivals corresponding to full triplets in the data. For the next six arrivals a window radius of $30 \mathrm{~ms}$ is taken since the time separation between adjacent peaks increases and, further, the lack of triplets in the data is accompanied with a larger variability of the peaks. For the remaining peaks the window radius is reduced to $20 \mathrm{~ms}$, which is in agreement with the decreasing separation of late arrivals.

The inversion results are described as follows. Figure 9 shows the identified acoustic peaks in the data (heavy lines) and also the locations of the peak arrivals predicted from the model (light lines). The gaps in the identified peak tracks are due to poor receptions in which the tomography signal is overwhelmed by the background noise at the receiver (cf. Fig. 5). These are demanding conditions for the automatic identification and tracking scheme since in the absence of peaks close to the predicted arrival times misidentifications are highly probable, which in turn may cause the subsequent inversions to lose track of the correct peaks.

The selected background states along with the retrieved EOF amplitudes are shown in Fig. 10. The seasonal trend is clear in both the selection of the background states as well as in the evolution of the EOF-1

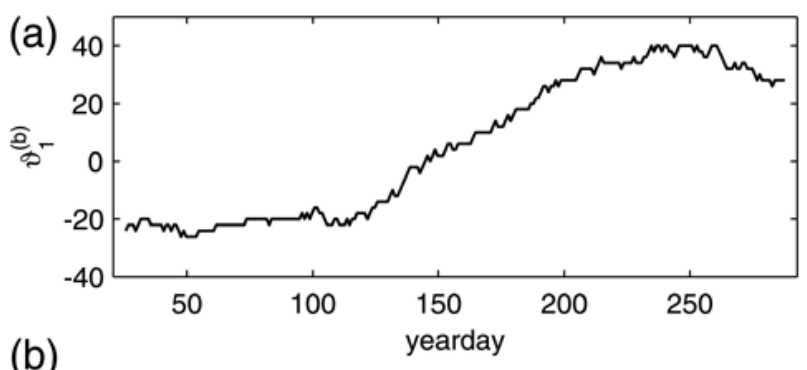

(b)

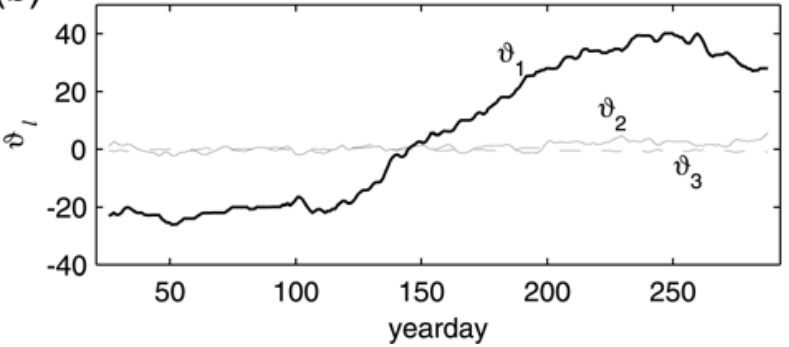

FIG. 10. (a) Estimated background states. (b) Estimated EOF amplitudes.

amplitude. Note that the background state is selected to account for the gross sound-speed variability responsible for the nonlinear traveltime behavior, whereas the fine tuning of the data is achieved through linear inversion about the selected background state. It is important to note that the variance of $\vartheta_{1}$ was a priori determined only in the finescale through the discretization of the parameter space. The large-scale variability, shown in Fig. 10, is determined from the tomography data.

In Fig. 11 the evolution of the depth-averaged temperatures is shown over three layers: the surface layer, from the surface to $150-\mathrm{m}$ depth; the intermediate layer, from 150- to 600-m depth, in which the mesoscale activity is expected to take place; and a layer from the surface to 2000-m depth, representing the total heat content of the water column. Note that the results represent averages over the $600-\mathrm{km}$ section. The heavy solid lines show the inversion results, whereas the rms errors are given through the shaded areas; these represent the cumulative error due to the linear inversion, the background state, and the identification function uncertainty, as well as the error of the sound speed-temperature conversion relation (see appendix B). Parallel to the tomography measurements, XBT data were systematically collected along the $\mathrm{W} 3-\mathrm{H}$ section. These data and also the CTD data from the deployment and recovery cruises are shown in Fig. 11 as crossed circles and squares, respectively. The dashed lines in Fig. 11 represent the climatological mean conditions.

Within the error bars, the inversion generally recovers the absolute temperatures and the trends in all three layers. A strong seasonal signal is present in the surface layer, whereas there appears to be no typical seasonal trend below $150 \mathrm{~m}$ - the variability observed below 150 $\mathrm{m}$ is mainly due to mesoscale activity. The comparison 


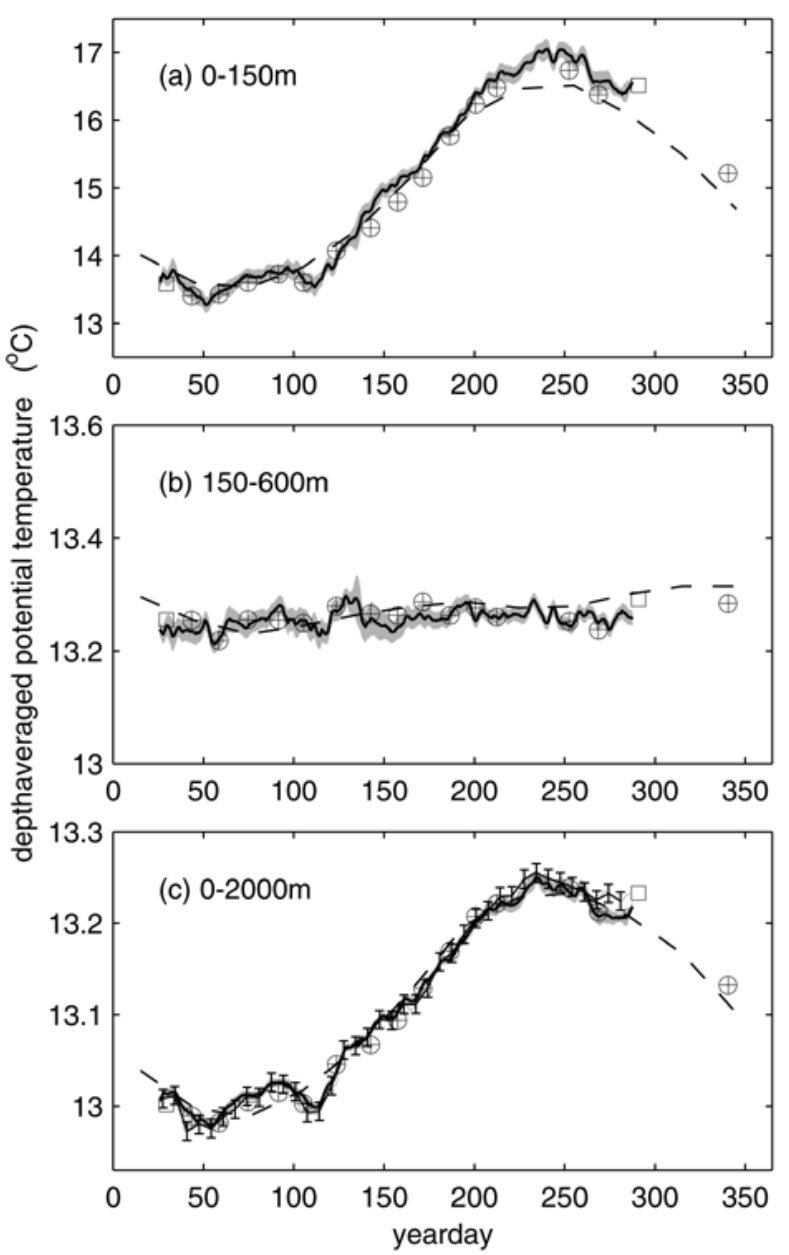

FIG. 11. Evolution of the depth-averaged potential temperatures in the (a) 0-150-, (b) 150-600-, and (c) 0-2000-m layers. The inversion results (heavy solid lines) and rms inversion errors (shaded areas) are compared with CTD data $(\square)$ from the Poseidon/Suroit cruises, with independent XBT data $(\oplus)$, and also with historical monthly mean temperatures (dashed lines). In the bottom panel results from single-ray calculations are shown (Send et al. 1997; light solid line) along with the corresponding error bars.

of the inversions with the climatological data shows that there are deviations from the climatological mean conditions in all three layers and this is also confirmed by the XBT data. The amplitude of the seasonal signal is $10 \%-20 \%$ larger in the observations than in the historical data. In addition there are a number of short-term or mesoscale fluctuations. The XBT data lie within the estimated error limits of the inversion, with the exception of a few points in the upper layer where the spatial variations may not be fully sampled by the XBT sections.

The light solid line and error bars in the bottom panel (0-2000 layer) of Fig. 11 represents heat content results from single-ray calculations (Send et al. 1997). A single early arrival was analyzed with that method, which sampled most of the water column vertically. Its propagation time was related to the water column heat content

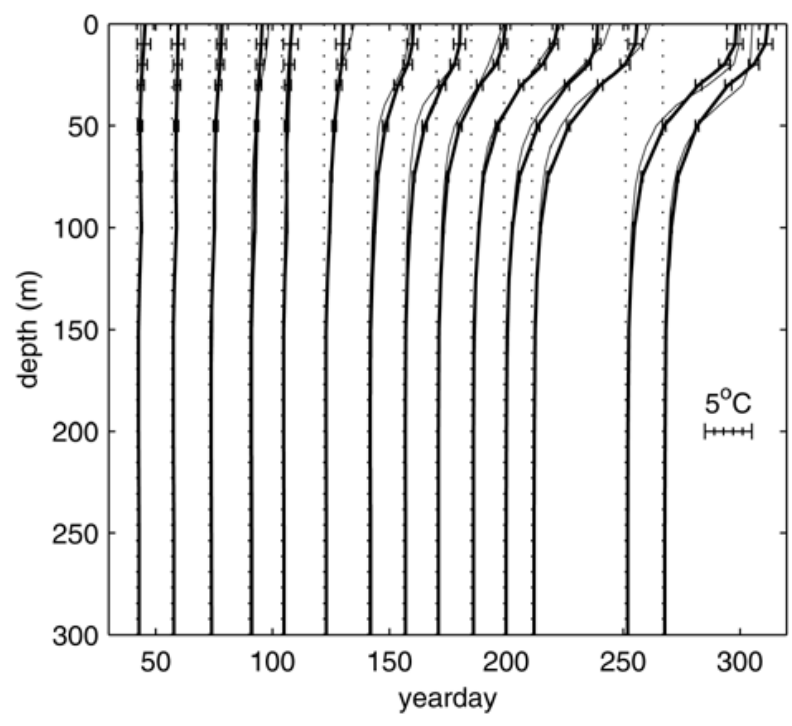

FIG. 12. Evolution of temperature profiles (deviations from $13.03^{\circ} \mathrm{C}$ ) in the upper $300-\mathrm{m}$ layer. Inversion results (heavy lines) and corresponding errors are compared with the range-averaged XBT temperature profiles (light lines).

through a large number of acoustic propagation simulations, using different historical states for the stratification, as well as perturbations corresponding to various oceanographic processes (convection, mesoscale eddies, mixed layer anomalies). The ensemble of all simulations was used to establish a single nonlinear relation between traveltime and heat content, including error estimates resulting from the scatter in the relation. The heat content time series obtained with this very different approach is further verification of the present inversion results.

Figure 12 shows the evolution of temperature profiles (deviations from $13.03^{\circ} \mathrm{C}$ ) in the upper $300 \mathrm{~m}$ comparing tomographic inversions (heavy lines) with the XBT measurements (light lines). The retrieved profiles are averaged profiles over the time window of each XBT sample. The inversion errors are also marked in Fig. 12. The seasonal temperature variability reaches $13^{\circ} \mathrm{C}$ at the surface and nearly disappears below 150-m depth. Also note that deviations between XBT data and inversions are larger in the surface layer than in the deeper layers, and this is in agreement with the inversion errors shown. The physical reason for the reduced tomography sensitivity near the sea surface lies in the particular temperature and sound-speed conditions in the Mediterranean, which give rise to either surface-reflected propagation in winter or channeled propagation with a shallow axis in summer. In both cases a very small fraction of the propagation time is spent in the near-surface layer, resulting in a poorer acoustic sampling of smaller depths.

\section{Discussion and conclusions}

In the present work a method is proposed extending the applicability of linear traveltime tomography to the 
case of large sound-speed variations and nonlinear traveltime behavior. The particular approach considers the background state as an unknown to be estimated from the tomography data, together with the identification function and the model (sound speed) parameters. For this purpose the sound-speed parameter space is discretized in accordance with the anticipated variability and degree of nonlinearity. Using the maximum likelihood approach and the Gaussian assumption the statistical estimation problem reduces to a problem of weighted least squares to be solved simultaneously for the three unknown quantities. A posteriori inversionerror estimates are obtained accounting for the uncertainty in the inversion, the background selection, and the peak identification.

The results of the demonstration case presented here are encouraging. The agreement with the independent XBT data is reasonable and consistent with the derived error estimates, thus also giving credibility to the technique of estimating the error size. Further, the heat content results from the present method were found to be compatible with those from the single-ray calculations by Send et al. (1997), which is an entirely different inversion approach.

The proposed scheme is generic and thus independent of the particular modeling approach for the propagation problem, either ray or wave theoretic, and the definition of observable arrival times; that is, it can apply equally well to ray, modal, or peak arrivals. Nevertheless, the computational burden and the efficiency of the overall method will be affected by the selection of the modeling approach. The steps to be undertaken for implementing the method are the following.

1) Definition and discretization of the parameter space in accordance with the anticipated variability (e.g., from the climatology) and degree of nonlinearity. The latter should be assessed from systematic direct runs-seasonal changes do not necessarily mean strong nonlinearities.

2) Solution of the direct problem for the different background states, association of peak arrivals between the different states and calculation of the background arrival times and observation matrices.

3) Application to the tomography data for the estimation of the background state, the identification function, and the model parameters.

The performance of the method depends on the quality of the tomography data. In the case of poor receptions, in which the tomographic signal is corrupted by noise, misidentifications may occur and this may have a misleading effect for the identifications in the subsequent receptions as well since the identification procedure makes use of previous-step results. In such cases, tuning of the search parameters and the observation errors may improve the situation. Alternatively, a preliminary analysis can be performed using the more robust part of the signal, for example the early arrivals, which is then used to constrain the complete analysis. In addition, work is in progress for solving the identification problem separately for each reception, thus minimizing the dependence on the previous-step results.

The proposed adaptive inversion scheme offers an alternative to linear inversion schemes based on an a priori selected time-variable background state. It is expected to be useful for the analysis of nonlinear traveltime tomography data, particularly in connection with broadband wave-theoretic modeling approaches, in which case other approaches such as iterative schemes would require a much larger computational burden. It may also be useful for the implementation of iterative schemes by providing favorable background states, based on actual receptions, to start iterations.

Acknowledgments. The present work was supported by the European Union under project MAS2-CT910006/Thetis-2. The authors would like to thank the anonymous reviewers for their helpful comments.

\section{APPENDIX A}

\section{Range-Dependence Effects}

The inversions performed in section $4 \mathrm{c}$ are range independent, yet the effects of range dependence on the late arrivals, especially on the cutoff peak, are significant and have to be taken into account. Using the Poseidon and Suroit sound-speed data it was seen in section $4 \mathrm{~b}$ that the range-dependent calculation predicts a cutoff peak, which is significantly delayed with respect to the range-independent prediction by $75-120 \mathrm{~ms}$. If this time delay is a stable feature throughout the expected range of variability, one could use this information in a quantitative way to reduce the discrepancy between the range-independent prediction and the observed data (Munk and Wunsch 1985).

The main range-dependent trend along $\mathrm{W} 3-\mathrm{H}$ is the north-south warming gradient shown in Fig. 2, which reaches an average of $10 \mathrm{~m} \mathrm{~s}^{-1}$ at the surface between $\mathrm{H}$ and W3. This trend is significant in the upper 200-m layer, whereas it is very weak below $250 \mathrm{~m}$. A simple model to describe this behavior is a linear range mode multiplied by a depth mode $\psi(z)$, which is zero below $250 \mathrm{~m}$. Figure A1 shows these two modes (heavy lines) compared with the actual range-dependent anomalies from the Poseidon/Suroit sound-speed data in normalized form; for the range-mode comparisons (top panel) the sound-speed data have been depth averaged over the layer from 0 to $150 \mathrm{~m}$.

To assess the effect of range dependence on the cutoff peak for the different background states, the above simple range-dependent model is superposed on each rangeindependent background environment. Thus, a set of synthetic range-dependent sections is generated resembling the conditions expected during the experiment:

$$
c(r, z)=c_{b}(z)+C(r-R / 2) \psi(z),
$$



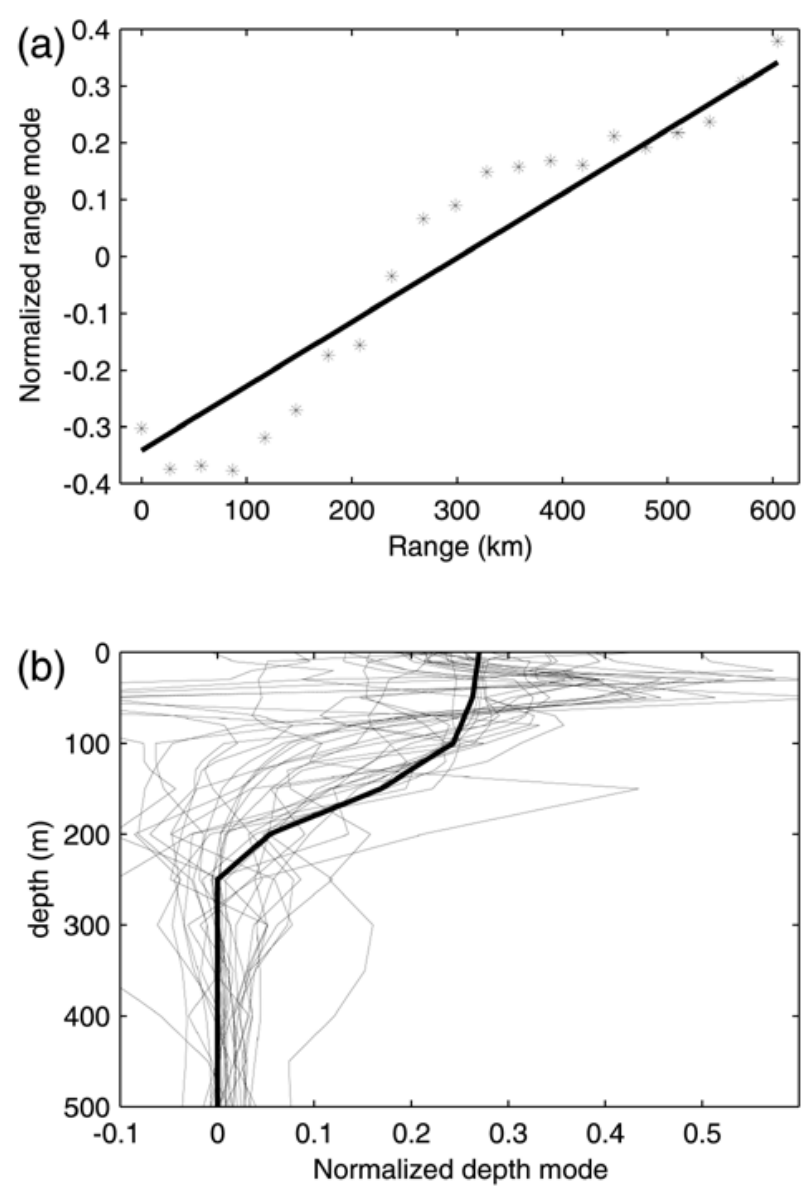

FIG. A1. (a) Linear normalized range mode (heavy line) compared with the normalized range anomaly $(*)$ of the Poseidon/Suroit soundspeed data, depth averaged in the upper $150 \mathrm{~m}$. (b) Normalized depth mode (heavy line) and normalized deviations (light lines) of the Poseidon/Suroit range-dependent sound-speed profiles from the corresponding range-averaged profiles.

where $r$ is the horizontal distance from the source and $R$ the source-receiver range. The constant $C$ is chosen such that the sound-speed difference at the surface between $\mathrm{H}$ and $\mathrm{W} 3$ is $10 \mathrm{~m} \mathrm{~s}^{-1}$, that is, $C R \psi(0)=10 \mathrm{~m}$ $\mathrm{s}^{-1}$ to resemble actual conditions. The range-dependent component $(r-R / 2) \psi(z)$ in (A1) has a zero range average, such that the synthetic range-averaged condition is described by the background profile $c_{b}(z)$.

Range-dependent/independent arrival-pattern predictions are performed in the following for $\vartheta_{1}=-40$, $-30, \ldots, 40$, using the KRAKEN normal/coupledmode code, assuming the emitted signal to be a Gaussian pulse with a central frequency of $250 \mathrm{~Hz}$ and a bandwidth of $62.5 \mathrm{~Hz}$. The results are shown in Fig. A2. The prediction in both the range-independent and rangedependent case is restricted to the first 20 propagating modes corresponding to the intermediate and late arrival patterns since this part is of interest here. Light lines represent the range-independent predictions and heavy lines, the range-dependent ones. Figure A2 confirms that

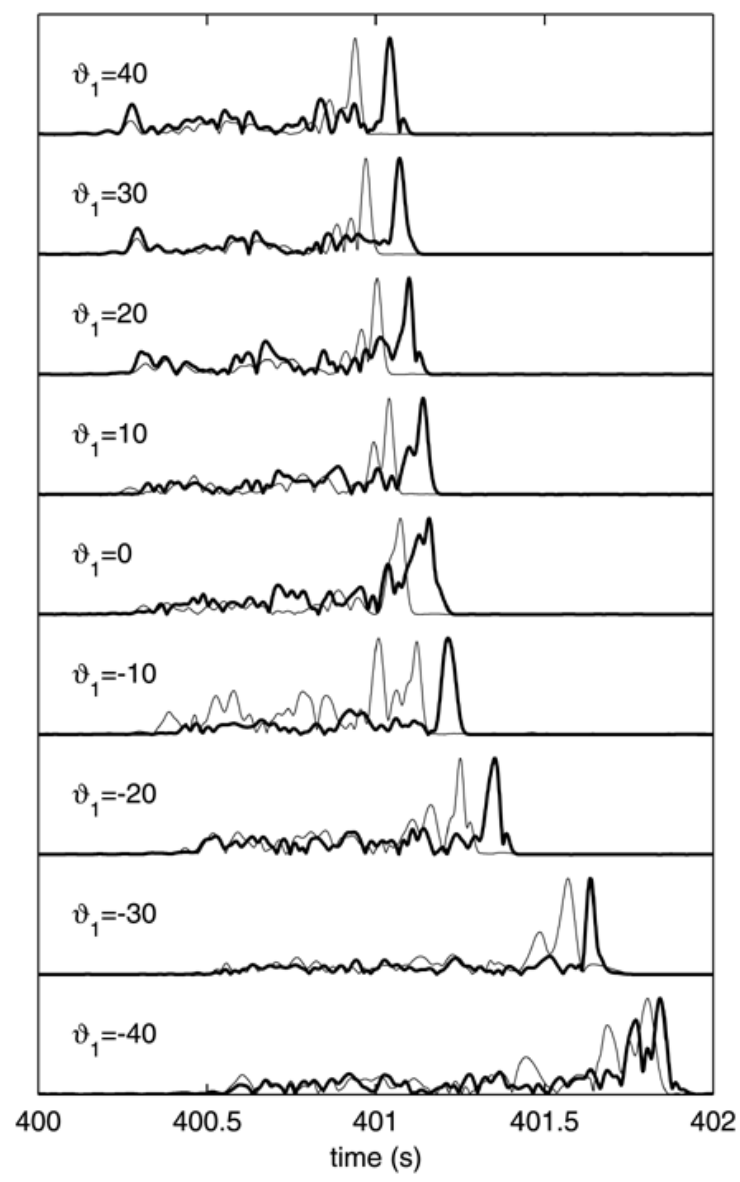

FIG. A2. Range-independent (light lines) and range-dependent (heavy lines) arrival pattern predictions for $\vartheta_{1}=-40,-30, \ldots$, 40.

there is a systematic time delay of the cutoff peak due to range dependence throughout the variability range of $\vartheta_{1}$.

Figure A3 shows the time delay of the cutoff peak as a function of $\vartheta_{1}$. The delay increases with increasing $\vartheta_{1}$ and ranges from $\delta \tau=80 \mathrm{~ms}\left(\vartheta_{1}=-40\right)$ to $\delta \tau=$ $140 \mathrm{~ms}\left(\vartheta_{1}=40\right)$. This trend and also the absolute delay values are compatible with the conclusions from the Poseidon/Suroit direct runs. The mean time delay is $100 \mathrm{~ms}$ and this is an estimate for the range-dependence effect on the cutoff peak anticipated during the experiment.

Figure A4 shows the range-dependence effect on the last 12 peak arrivals used for inversions. Three cases are considered for the range-averaged profile $\left(\vartheta_{1}=40\right.$, $0,-40)$, which span the anticipated seasonal variability. In order to track the peaks the range-dependent anomaly is gradually increased from zero (range-independent case) to the anticipated extent, reaching $10 \mathrm{~m} \mathrm{~s}^{-1}$ at the surface. Due to the finite bandwidth of the emitted signal, nearby peaks interact with each other and this contributes to the nonsmoothness of the tracks in Fig. A4. The range-dependence effect is strongest for the cutoff 


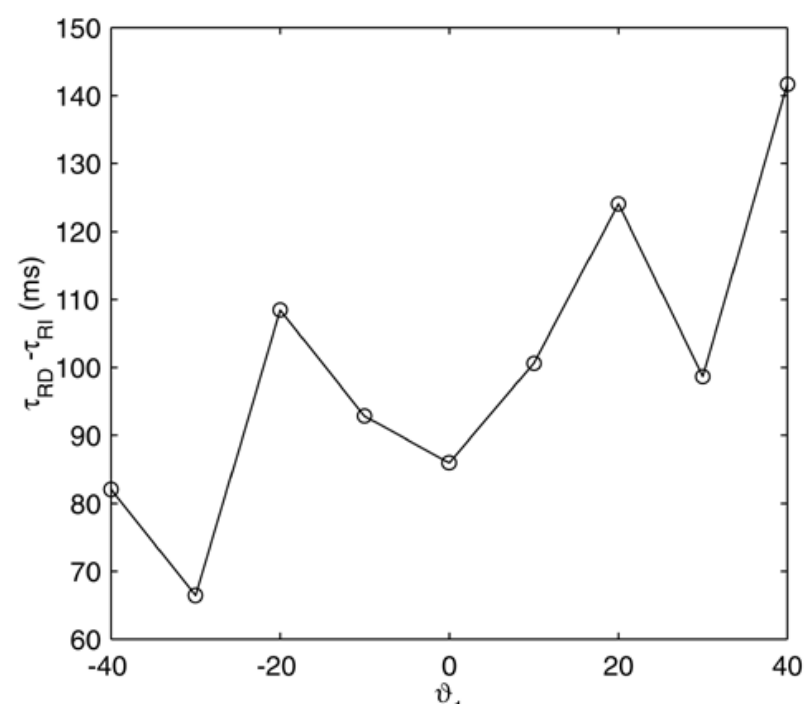

FIG. A3. Delay of the cutoff peak due to a fixed range dependence (product of the range and depth modes shown in Fig. A1), as a function of $\vartheta_{1}$.

peak, which is in agreement with the results shown in Fig. A3. For the earlier peaks, separated from the cutoff peak by more than $250 \mathrm{~ms}$, the effect is significantly weaker and lies within the rms error used for the rangeindependent inversions $(20 \mathrm{~ms}$ for the first 3 peaks and $30 \mathrm{~ms}$ for the remaining 8 peaks). The overall rangedependent effect becomes weaker with decreasing $\vartheta_{1}$, which corresponds to upward-refracted, surface-reflected propagation along the entire $\mathrm{W} 3-\mathrm{H}$ section. In conclusion, after correcting for the systematic range-dependent effect on the cutoff peak, the range-independent description can be used for performing inversions.

\section{APPENDIX B}

\section{Conversion of Sound Speed to Temperature}

Some care should be applied in converting sound speed, which is the result of the inversions, to temperature. In order to construct an appropriate relation with pertinent error bars, sound-speed formulas were combined with historical data in the area of the experiment. First, the average relation between sound speed and temperature was obtained as a function of depth, which included the effect of the mean salinity at each level. The conversion factor thus obtained ranged from about $0.35^{\circ} \mathrm{C} \mathrm{s} \mathrm{m}^{-1}$ near the surface to $0.27^{\circ} \mathrm{C} \mathrm{s} \mathrm{m}^{-1}$ at $2000-\mathrm{m}$ depth. Since salinity also has an effect on sound speed, this introduces an uncertainty into the conversion. Using the salinity variability at each level from the historical dataset, its effect on the temperature estimate based on sound speed was calculated. It results in a strong function of depth, with rms temperature uncertainty ranging from $0.1^{\circ}-0.2^{\circ} \mathrm{C}$ in the upper $100 \mathrm{~m}$ to $0.005^{\circ} \mathrm{C}$ in the lower layers. For the inversions presented in the main section of the paper, the depth-dependent sound speed- (a)

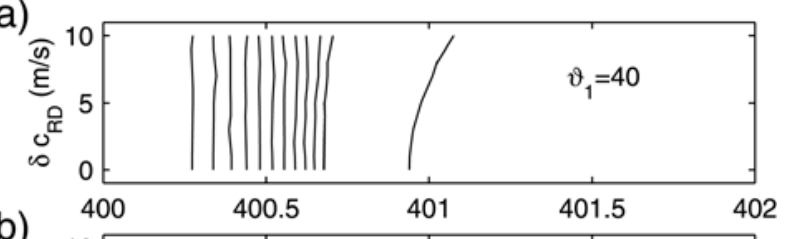

(b)

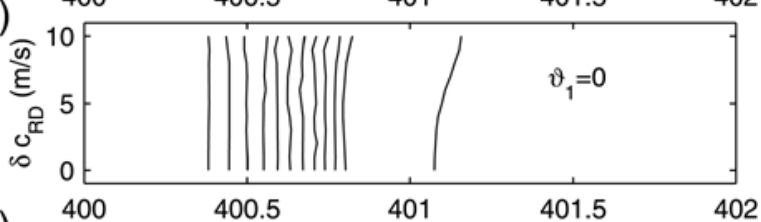

(c)

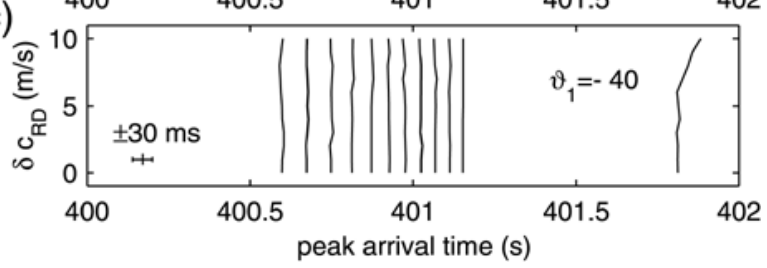

FIG. A4. The arrival times of the last 12 peak arrivals used for the inversion as functions of the range-dependence intensity (denoted on the vertical axis through the range-dependent anomaly at the surface) for $\vartheta_{1}=$ (a) 40, (b) 0 , and (c) -40 .

temperature conversion was used, while the uncertainty from salinity entered the error estimates of the temperature inversions.

\section{REFERENCES}

Aki, K., and P. G. Richards, 1980: Quantitative Seismology. Theory and Methods. Vol. 1. W. H. Freeman and Company, 557 pp.

Athanassoulis, G. A., and E. K. Skarsoulis, 1995: Arrival-time perturbations of broadband tomographic signals due to sound-speed disturbances. A wave-theoretic approach. J. Acoust. Soc. Amer., 97, 3575-3588.

Cornuelle, B., and Coauthors, 1985: Tomographic maps of the ocean mesoscale. Part I: Pure acoustics. J. Phys. Oceanogr., 15, 133152.

—_, and Coauthors, 1993: Ocean acoustic tomography at 1000-km range using wavefronts measured with a large-aperture vertical array. J. Geophys. Res., 98, 16 365-16 377.

Desaubies, Y., 1990: Ocean acoustic tomography. Oceanographic and Geophysical Tomography, Y. Desaubies, A. Tarantola, and J. Zinn-Justin, Eds., Elsevier, 159-202.

Dushaw, B. D., P. F. Worcester, B. D. Cornuelle, and B. M. Howe, 1993: Variability of heat content in the central North Pacific in summer 1987 determined from long-range acoustic transmissions. J. Phys. Oceanogr., 23, 2650-2666.

Franklin, J. N., 1970: Well-posed stochastic extensions of ill-posed linear problems. J. Math. Anal. Appl., 31, 682-716.

GSP Group, 1990: Greenland Sea Project-A venture toward improved understanding of the ocean's role in climate. Eos, Trans. Amer. Geophys. Union, 71, 750-751; 754-755.

Jones, R. M., T. M. Georges, and J. P. Riley, 1986: Inverting verticalslice tomography measurements for asymmetric ocean soundspeed profiles. Deep-Sea Res., 33, 601-619.

—, E. C. Shang, and T. M. Georges, 1993: Nonperturbative modal tomography inversion. Part I. Theory. J. Acoust. Soc. Amer., 94, 2296-2302.

_ B. M. Howe, J. A. Mercer, R. C. Spindel, and T. M. Georges, 1994: Nonperturbative ocean acoustic tomography inversion of $1000-\mathrm{km}$ pulse propagation in the Pacific Ocean. J. Acoust. Soc. Amer., 96, 3054-3063. 
Marshall, J., and Coauthors, 1998: The Labrador Sea deep convection experiment. Bull. Amer. Meteor. Soc., 79, 2033-2058.

Mauuary, D., 1994: Detection, estimation et identification pour la tomographie acoustique oceanique. Étude theorique et experimentale. Ph.D dissertation, Institut National Polytechnique de Grenoble, 165 pp. [Available from INPG/ENSIEG, BP 4638402 , St. Martin d'Hères, Grenoble, France.]

Menemenlis, D., T. Webb, C. Wunsch, U. Send, and C. Hill, 1997a: Basin-scale ocean circulation from combined altimetric, tomographic and model data. Nature, 385, 618-621.

- D. Stammer, C. Wunsch, B. D. Dushaw, and the ATOC Group, 1997b: Preliminary estimates of North Pacific circulation from combined altimetry, acoustic tomography, and a general circulation model. Int. WOCE Newslett., 25, 7-10.

Mercer, J. A., and J. R. Booker, 1983: Long-range propagation of sound through oceanic mesoscale structures. J. Geophys. Res. 88, 689-699.

Munk, W. H., 1994: Heard Island and beyond. J. Acoust. Soc. Amer., 95, 567.

— 1996: Acoustic thermometry of ocean climate. J. Acoust. Soc. Amer., 100A, 2580.

- C. Wunsch, 1979: Ocean acoustic tomography: A scheme for large-scale monitoring. Deep-Sea Res., 26A, 123-161.

$\longrightarrow$, and 1983: Ocean acoustic tomography: Rays and modes. Rev. Geophys. Space Phys., 21, 777-793.

$\longrightarrow$, and - 1985: Biases and caustics in long-range acoustic tomography. Deep-Sea Res., 32, 1317-1346.

— , and A. M. G. Forbes, 1989: Global ocean warming: An acoustic measure? J. Phys. Oceanogr., 19, 1765-1778.

— , P. F. Worcester, and C. Wunsch, 1995: Ocean Acoustic Tomography. Cambridge University Press, 433 pp.

Ocean Tomography Group, 1982: A demonstration of ocean acoustic tomography. Nature, 299, 121-125.

Pawlowicz, R., J. F. Lynch, W. B. Owens, P. F. Worcester, W. M. L. Morawitz, and P. J. Sutton, 1995: Thermal evolution of the Greenland Sea Gyre in 1988-1989 J. Geophys. Res., 100, $4727-$ 4750 .
Porter, M. B., and E. L. Reiss, 1984: A numerical method for ocean acoustic normal modes. J. Acoust. Soc. Amer., 76, 244-252.

Send, U., 1996a: Peak tracking by simultaneous inversion: Toward a one-step acoustic tomography analysis. J. Atmos. Oceanic Technol., 13, 1116-1122.

— Ed., 1996b: A pilot tomography system for monitoring the western Mediterranean basin. EU MAST-2 Project Final Rep. CT91-0006, 177 pp. [Available from U. Send, Institut für Meereskunde-Kiel, Düsternbrooker Weg 20, 24105 Kiel, Germany.]

_, F. Schott, F. Gaillard, and Y. Desaubies, 1995: Observation of a deep convection regime with acoustic tomography. J. Geophys. Res., 100, 6927-6941.

— , and Coauthors, 1997: Acoustic observations of heat content across the Mediterranean Sea. Nature, 385, 615-617.

Skarsoulis, E. K., 1996: An adaptive scheme for ocean acoustic tomography of large sound-speed variations. Proc. Third European Conf. on Underwater Acoustics, Heraklion, Crete, Greece, European Commission/European Acoustics Association, 803-808.

— , G. A. Athanassoulis, and U. Send, 1996: Ocean acoustic tomography based on peak arrivals. J. Acoust. Soc. Amer., 100, 797-813.

Spiesberger, J. L., 1985: Gyre-scale acoustic tomography: Biases, iterated inversions, and numerical methods. J. Geophys. Res., 90, 869-876.

— J. Acoust. Soc. Amer., 89, 2677-2688.

— themometers. Oceanography, 5, 92-98.

,-- , and J. A. Furgerson, 1992: Listening for climatic temperature change in the northeast Pacific: 1983-1989. J. Acoust. Soc. Amer., 92, 384-396.

- H. E. Hurlburt, M. Johnson, M. Keller, S. Meyers, and J. O'Brien, 1998: Acoustic thermometry data compared with two ocean models: The importance of Rossby waves and ENSO in modifying the ocean interior. Dyn. Atmos. Oceans, 26, 209-240.

Tarantola, A., 1987: Inverse Problem Theory. Elsevier, 613 pp.

Worcester, P. F., and Coauthors, 1993: Evolution of the large-scale temperature field in the Greenland Sea during 1988-1989 from tomographic measurements. Geophys. Res. Lett., 20, 2211-2214. 